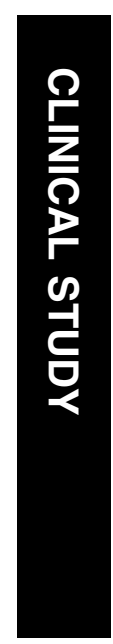

\section{Assessment of macular function by microperimetry in unilateral resolved central serous chorioretinopathy}

H Ozdemir ${ }^{1}$, SA Karacorlu' ${ }^{1}$, F Senturk ${ }^{1}$, M Karacorlu' ${ }^{1}$ and O Uysal ${ }^{2}$
'Ophthalmology, The Istanbul Retina Institute Inc., Unimed Center, Istanbul, Turkey

${ }^{2}$ Department of Biostatistics, Cerrahpasa School of Medicine, University of Istanbul, Istanbul, Turkey

Correspondence:

M Karacorlu,

Ophthalmology, Istanbul Retina Institute Inc., Unimed Center, Hakkı Yeten Cad., No.: 8/7, Fulya, Sisli 34349, Istanbul, Turkey

Tel: + 902122313121

Fax: + 902122332425

E-mail: retina@pobox.com

Received: 16 January 2006 Accepted in revised form: 5 July 2006

Published online: 25 August 2006

The authors have no commercial interest in the material used in this work

\begin{abstract}
Purpose To determine macular sensitivity and fixation characteristics in patients with unilateral resolved central serous chorioretinopathy (CSC) using fundus-related microperimetry.

Methods We reviewed 15 eyes with resolved CSC and 15 normal healthy eyes that had undergone fundus-related microperimetry. The macular sensitivity was measured using the recently introduced fundus-related microperimeter, MP-1. The best-corrected visual acuity (VA) (BCVA), mean retinal sensitivity in the central $10^{\circ}$ (central microperimetry, cMP-1) and in the paracentral 10-20 (paracentral microperimetry, pMP-1), and fixation stability and location were determined and compared with measurements in control eyes.

Results BCVA at the time of this study was 20/20 in all the affected eyes, and fundus examination and optical coherence tomography findings revealed no serous detachment. Eyes with CSC showed statistically significantly lower cMP-1 sensitivity and lower, but not significantly, pMP-1 sensitivity than control eyes $(P<0.001$, $P=0.11$, respectively). Eyes with CSC were not significantly different from control eyes in fixation location $(P=1.00)$ or fixation stability $(P=0.91)$. Fixation location was predominantly central in all eyes with CSC; fixation was stable in $12(80 \%)$ and relatively unstable in $3(20 \%)$.

Conclusion Our study shows that eyes with resolved CSC can have lower retinal sensitivity in the central macula than control eyes, even after good VA has been obtained. Eye (2008) 22, 204-208; doi:10.1038/sj.eye.6702563; published online 25 August 2006
\end{abstract}

Keywords: central serous chorioretinopathy; retinal sensitivity; fundus-related microperimetry; macular function; fixation stability; fixation location

Introduction

Central serous chorioretinopathy (CSC) is a condition characterized by idiopathic leaks from the retinal pigment epithelium (RPE) leading to serous retinal detachment. In the early phase of disease, visual acuity (VA) may be good despite the macular detachment. After spontaneous recovery, patients may have residual visual symptoms such as metamorphopsia or loss of contrast sensitivity, despite recovering normal VA. More chronic forms of CSC are associated with atrophic and degenerative changes of the retina and RPE and, consequently, with VA decline. ${ }^{1}$

Many studies have examined functional deficiencies of the detached retina in CSC. Even when VA is reasonably good there is compromise of functions such as contrast sensitivity, colour discrimination, dark adaptation, focal electroretinography (ERG), and microperimetry. ${ }^{2-6}$ This study was undertaken to examine the function of the macula in patients with unilateral resolved CSC. Microperimetry, a technique for accurately testing macular sensitivity and retinal fixation, with strict correspondence of visual parameters and macular morphology, was used for the examination of macular function.

\section{Methods}

We retrospectively reviewed cases of unilateral resolved CSC involving the fovea. Each patient 
had a documented episode of CSC in one eye and no CSC diagnosed or suggested in the fellow eye by any history of decreased vision, central scotoma, or central distortion. Only patients with no evidence of subretinal fluid under the macula for at least 2 months were evaluated in this study. Because several diseases may influence the microperimetry result, we excluded patients with lenticular and corneal opacities, a history of refractive surgery, glaucoma, or ocular hypertension, a history of intraocular inflammation such as anterior or posterior uveitis, multifocal choroiditis, a history of retinal detachment, a history of ocular trauma, and optic neuropathy. As it is very difficult to quantify accurately the duration of detachment for patients with CSC, this information was not collected. A medical and ocular history was obtained for each patient, and a complete ophthalmic examination, including determination of best-corrected visual acuity (BCVA), slit-lamp biomicroscopy, indirect ophthalmoscopy, fluorescein angiography, and optical coherence tomography (OCT), was performed. BCVA expressed as logMAR was obtained at a distance of $4 \mathrm{~m}$. Fluorescein angiogram was performed on a Heidelberg scanning laser ophthalmoscope (SLO) (Heidelberg Engineering, Heidelberg, Germany). All OCT examinations were carried out using the OCT 3000 scanner (Carl Zeiss Ophthalmic System Inc., Humphrey Division, Dublin, CA, USA). Macular sensitivity was evaluated by MP-1 microperimetry (MP-1, Nidek Technologies, Italy). The MP-1 available in June 2003 (Version: MP-1 SW 1.4.1 SP1) was used for microperimetry. The MP-1 provides a $45^{\circ}$ nonmydriatic view of the fundus with automated correction for eye movements. Goldmann III white stimuli and a 4-2 staircase strategy were used, and a circular test grid with 74 stimulus locations covering an area of $20^{\circ}$ was applied. The fixation target was $1^{\circ}$ red cross and for testing the contralateral eye was occluded. The mean retinal sensitivities at the 28 locations covering the central $10^{\circ}$ (central microperimetry, cMP-1) and at the 48 locations covering the paracentral $10-20^{\circ}$ (paracentral microperimetry, pMP-1) were determined.

The stimuli were projected on a white background with background luminance set to $1.27 \mathrm{~cd} / \mathrm{m}^{2}$ and a stimulus presentation time of $200 \mathrm{~ms}$. The perimetric strategy of the current software version of the MP-1 starts with an initially defined threshold level for each stimulus. A 4-2 staircase strategy is then carried out, and the last seen threshold value is taken as the final threshold. In addition, the instrument tests the same luminance levels at all test locations before moving on to the next luminance level (ie, for all locations one luminance level is projected after the other). Differential light threshold values were compared by calculating selected points, which were averaged automatically by the MP-1 microperimetry software program for mean sensitivity in a polygon. For the assessment of fixation, the fundus movements were tracked during examination, whereas the patient gazed at the fixation target. The autotracking system calculated horizontal and vertical shifts relative to a reference frame and drew a map of the patient's eye movements during the examination. The recorded fixation points were classified into three categories for fixation stability analysis (stable, relatively unstable, and unstable). Fixation was regarded as 'stable' if more than $75 \%$ of the fixation points were inside the $2{ }^{\circ}$ diameter circle, as 'relatively unstable' if less than $75 \%$ were inside the $2^{\circ}$ diameter circle but more than $75 \%$ inside the $4^{\circ}$ diameter circle, and as 'unstable' if less than $75 \%$ of the fixation points were inside the $4^{\circ}$ diameter circle. To assess fixation location, a standard, circular, central fixation area $2^{\circ}$ in diameter (approximately $700 \mu \mathrm{m}$ ) centred on the fovea was defined. The standard $2^{\circ}$ circle was placed by looking for the centre of the foveal avascular zone (FAZ). Eyes with more than $50 \%$ of the preferred fixation points located within the central fixation area were classified as having predominantly central fixation. Eyes with more than $25 \%$ but less than $50 \%$ of the preferred fixation points located within the central fixation area were classified as having poor central fixation. Eyes with less than $25 \%$ of the preferred fixation points located within the central fixation area were classified as having predominantly eccentric fixation. Fixation characteristics were classified automatically by the MP-1 microperimetry software, after a landmark had been positioned in the centre of the FAZ. Results from age-matched control eyes (age range, 24-46 years, mean age, 38 years) and eyes with resolved CSC were compared by Student's $t$-test and the Mann-Whitney U-test. Written informed consent was obtained from all subjects, and the study was conducted in accordance with the tenets of the Declaration of Helsinki Principle.

\section{Results}

Fifteen eyes of 15 patients with unilateral resolved CSC were evaluated in this series. There were 12 men and three women whose ages ranged from 24 to 47 years (mean age, 36 years). BCVA at the time of this study was $20 / 20(\log$ MAR $0.0( \pm 0.0))$ in all the affected eyes, and fundus examination, fluorescein angiography, and OCT findings revealed no serous detachment. There were some RPE alterations in the affected eyes. The clinical characteristics of CSC eyes are reported in Table 1. Figure 1 shows fluorescein angiography (A), and MP-1 microperimetry sensitivity and fixation properties (B) in patient number 14 . 
Table 1 Clinical characteristics of patients with CSC

\begin{tabular}{rccccc}
\hline Patient & $\begin{array}{c}\text { Age } \\
\text { (years) }\end{array}$ & $\begin{array}{c}\text { MP-1 } \\
\text { Microperimetry } \\
\text { sensitivity(dB) } \\
\text { cMP-1 }\end{array}$ & $\begin{array}{c}\text { MP-1 } \\
\text { Microperimetry } \\
\text { sensitivity(dB) } \\
\text { pMP-1 }\end{array}$ & $\begin{array}{c}\text { MP-1 } \\
\text { Microperimetry } \\
\text { fixation } \\
\text { location }^{\text {a }}\end{array}$ & $\begin{array}{c}\text { MP-1 } \\
\text { Microperimetry } \\
\text { fixation } \\
\text { stability }^{\mathrm{b}}\end{array}$ \\
\hline 1 & & 15.5 & 13.8 & 3 & 3 \\
2 & 40 & 16.1 & 14.5 & 3 & 3 \\
3 & 40 & 12.9 & 13.0 & 3 & 3 \\
4 & 46 & 14.4 & 12.5 & 3 & 3 \\
5 & 29 & 16.1 & 15.7 & 3 & 3 \\
6 & 29 & 16.1 & 12.1 & 3 & 3 \\
7 & 47 & 17.0 & 15.0 & 3 & 3 \\
8 & 47 & 14.9 & 18.2 & 3 & 3 \\
9 & 29 & 19.0 & 17.0 & 3 & 3 \\
10 & 24 & 13.1 & 14.2 & 3 & 3 \\
11 & 30 & 12.8 & 13.1 & 3 & 3 \\
12 & 33 & 12.7 & 13.7 & 3 & 3 \\
14 & 46 & 16.6 & 16.5 & 3 & 3 \\
15 & 43 & 15.4 & 15.3 & 3 & 3 \\
\hline
\end{tabular}

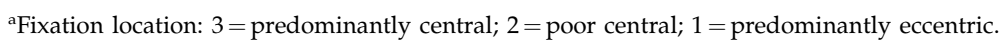

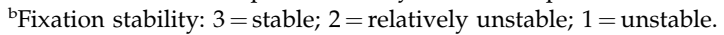

Table 2 Mean cMP-1 and pMP-1 retinal sensitivity, fixation location, and fixation stability in eyes with CSC and control eyes

\begin{tabular}{|c|c|c|c|c|}
\hline & Control eyes (mean $\pm S D)$ & Eyes with CSC (mean $\pm S D)$ & Statistic & P-value \\
\hline Sensitivity (cMP-1, dB) & $16.51 \pm 0.92$ & $11.75 \pm 2.45$ & $t=2.84$ & $<0.05$ \\
\hline Sensitivity (pMP-1, dB) & $15.56 \pm 1.22$ & $14.72 \pm 1.76$ & $t=1.65$ & 0.11 \\
\hline Fixation location (median) ${ }^{\mathrm{a}}$ & 3 & 3 & $z=0.00^{\mathrm{b}}$ & $>0.99$ \\
\hline Fixation stability (median) ${ }^{c}$ & 3 & 3 & $z=0.11^{\mathrm{b}}$ & 0.91 \\
\hline
\end{tabular}

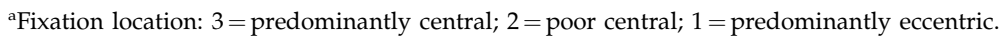

${ }^{\mathrm{b}}$ Mann-Whitney U-test.

${ }^{c}$ Fixation stability: $3=$ stable; $2=$ relatively unstable; $1=$ unstable.

The mean central and paracentral sensitivity, fixation location, and fixation stability in diseased and control eyes are shown in Table 2. Eyes with CSC showed statistically significantly lower cMP-1 sensitivity and lower, but not significantly, pMP-1 sensitivity than control eyes $(P<0.05, P=0.11$, respectively). Eyes with CSC were not significantly different from control eyes in fixation location $(P=1.00)$ or fixation stability $(P=0.91)$. Fixation location was predominantly central in all eyes with CSC; fixation was stable in $12(80 \%)$ and relatively unstable in $3(20 \%)$.

\section{Discussion}

Abnormalities of macular function have been shown in eyes with active CSC by subjective tests and also by objective methods such as focal macular ERG and multifocal electroretinography (mfERG). ${ }^{4,7,8}$ Toonen et $a l^{6}$ also found decreased retinal sensitivity in the affected areas with SLO microperimetry. These results reflect the wide range of visual and functional difficulties that have been found in serous detachments. ${ }^{2-3}$ These functional defects have generally been attributed to retinal separation, which affects the transport of nutrients and visual pigments and also may allow some disruption of photoreceptor orientation. ${ }^{7}$

However, evidence is increasing that in CSC pathologic changes occur over a larger area than the detachment itself, in which case some of the functional abnormalities may originate from underlying choroidal dysfunction. ${ }^{9}$ Vajaranant $e t \mathrm{al}^{8}$ showed that mfERG abnormalities were not limited to serous elevation. Marmor and $\operatorname{Tan}^{7}$ also showed that, according the mfERG findings, macular electrical function was abnormal beyond the area of detachment. These results support not only the reality of widespread choroidal dysfunction in CSC but also functional abnormalities in the reattached retina. Experimental animal models have shown that retinal detachment and reattachment can induce a variety of cellular changes that may account for decreased VA. ${ }^{10-12}$ Retinal detachment can cause photoreceptor cell apoptosis as early as 1-3 days after 

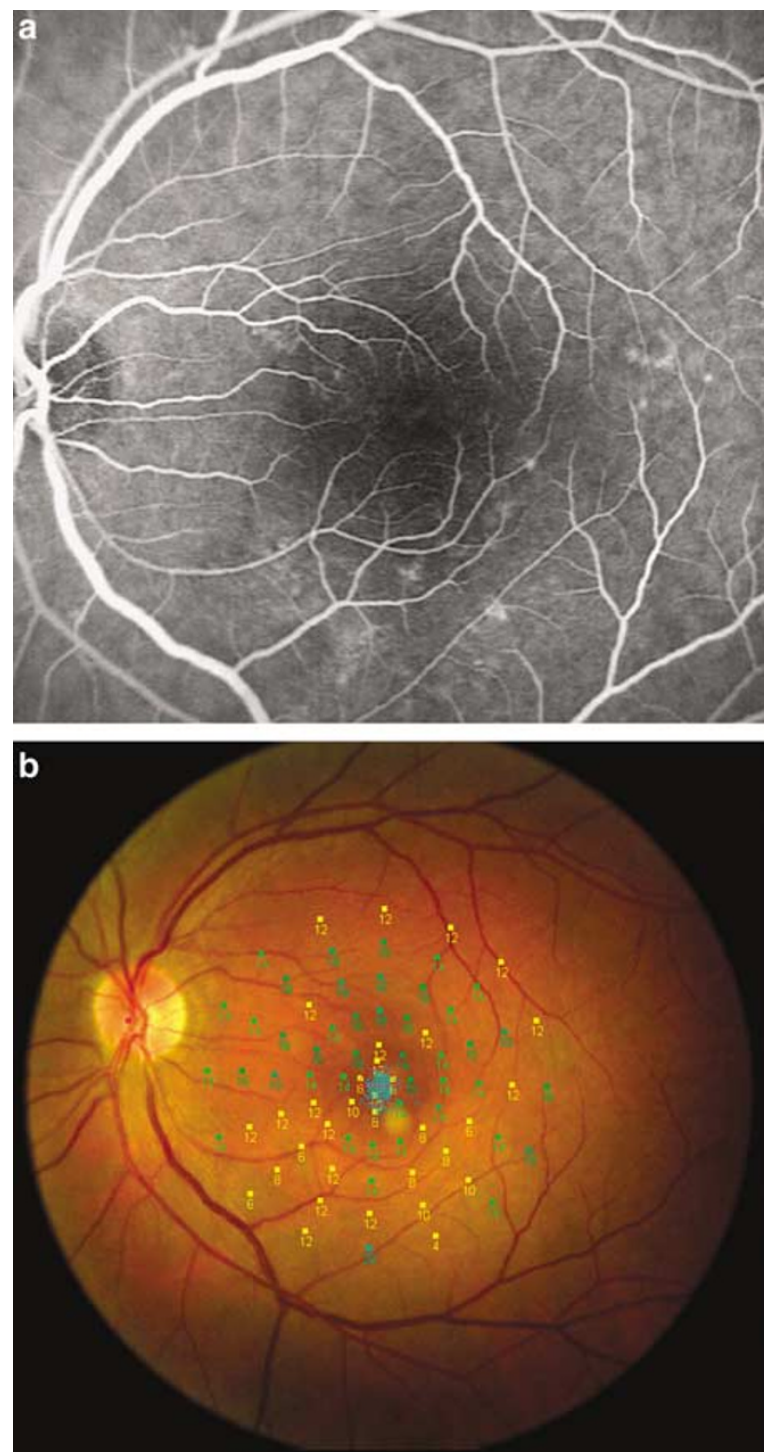

Figure 1 Fluorescein angiography (a), and MP-1 microperimetry sensitivity and fixation properties (b) in patient number 14 .

retinal detachment. ${ }^{13,14}$ Loss of photoreceptor outer segments is also a common finding after detachment. ${ }^{10,11}$ Other changes include hypertrophy and hyperplasia of Müller cells, which can invade the subretinal space and form a barrier to RPE-photoreceptor reapposition. ${ }^{11-15}$ With reattachment, the retina can recover to some degree, but this recovery occurs in a patchwork of morphologic abnormalities including a decreased number of cells in the outer nuclear layers. ${ }^{16}$ Functional abnormalities can persist in the reattached rabbit retina, even after a brief retinal detachment followed by retinal reattachment. ${ }^{17}$ Although there is not a good experimental model of CSC, and experimental rhegmatogenous retinal detachment might not emulate all aspects, it is possible that the observation we made with microperimetry in unilateral resolved CSC may correlate with the alterations seen by histologic analysis in animal models of retinal detachment. Detachment caused by CSC may lead to varying amounts of photoreceptor loss, atrophy, and shortening of photoreceptor outer segments. These changes may have led to the observation of the decrease in retinal sensitivity in the central macular area even after active CSC was resolved and even after good VA was obtained.

In this study, fixation characteristics (location and stability) were also determined. It is well known that important daily tasks, such as recognition of symbols, orientation, and reading, are strongly dependent on fixation stability and fixation location. ${ }^{18-20}$ Like other macular disorders, CSC can affect fixation properties. According to our results, eyes with resolved CSC are not significantly different from control eyes in fixation location and fixation stability. In diseased eyes, fixation localization was predominantly central in all patients and fixation stability was stable in 12 out of 15 patients. This means that even though the retinal sensitivity was decreased, our patients had central and stable fixation in their affected eyes.

This study, with a retrospective design and a small sample size, has numerous limitations. The functional changes as described in this article may be dependent on the duration of the retinal detachment; however, we do not know of an accurate way to quantify the duration in affected patients. Despite these limitations, our study shows that eyes with resolved CSC have lower retinal sensitivity in the central macula than control eyes, even after good VA has been obtained.

\section{References}

1 Spaide RF, Campesas L, Haas A, Yannuzzi LA, Fisher YL, Guyer DR et al. Central serous chorioretinopathy in younger and older adults. Ophthalmology 1996; 103: 2070-2080.

2 Folk JC, Thompson HS, Han DP, Brown CK. Visual function abnormalities in central serous retinopathy. Arch Ophthalmol 1984; 102: 1299-1302.

3 Chuang EL, Sharp DM, Fitzke FW, Kemp CM, Holden AL, Bird AC. Retinal dysfunction in central serous retinopathy. Eye 1987; 1: 120-125.

4 Miyake Y, Shiroyama N, Ota I, Horiguchi M. Local macular electroretinographic responses in idiopathic central serous chorioretinopathy. Am J Ophthalmol 1988; 106: 546-550.

5 Nagata M, Honda Y. Macular ERG in central serous retinopathy. Jpn J Ophthalmol 1971; 15: 9-16.

6 Toonen F, Remky A, Janssen V, Wolf S, Reim M. Microperimetry in patients with central serous retinopathy. Ger J Ophthalmol 1995; 4: 311-314.

7 Marmor MF, Tan F. Central serous chorioretinopathy: bilateral multifocal ERG abnormalities. Arch Ophthalmol 1999; 117: 184-188.

8 Vajaranant TS, Szlyk JP, Fishman GA, Gieser JP, Seiple W et al. Localized retinal dysfunction in central serous 
chorioretinopathy as measured using the multifocal electroretinogram. Ophthalmology 2002; 109: 1243-1250.

9 Marmor MF. On the cause of serous detachment and acute central serous chorioretinopathy. Br J Ophthalmol 1997; 81: 812-813.

10 Kroll AJ, Machemer R. Experimental retinal detachment in the owl monkey. III. Electron microscopy of retina and pigment epithelium. Am J Ophthalmol 1968; 66: 410-427.

11 Anderson DH, Guerin CJ, Erickson PA, Stern WH, Fisher SK et al. Morphological recovery in the reattached retina. Invest Ophthalmol Vis Sci 1986; 27: 168-183.

12 Rex TS, Fariss RN, Lewis GP, Linberg KA, Sokal I, Fisher SK A survey of molecular expression by photoreceptors after experimental retinal detachment. Invest Ophthalmol Vis Sci 2002; 43: 1234-1247.

13 Cook B, Lewis GP, Fisher SK, Adler R. Apoptotic photoreceptor degeneration in experimental retinal detachment. Invest Ophthalmol Vis Sci 1995; 36: 990-996.

14 Hisatomi T, Sakamoto T, Goto Y, Yamanaka I, Oshima Y, Hata $Y$ et al. Critical role of photoreceptor apoptosis in functional damage after retinal detachment. Curr Eye Res 2002; 24: 161-172.
15 Lewis GP, Fisher SK. Muller cell outgrowth after retinal detachment: association with cone photoreceptors. Invest Ophthalmol Vis Sci 2000; 41: 1542-1545.

16 Sakai T, Calderone JB, Lewis GP, Linberg KA, Fisher SK, Jacobs GH. Cone photoreceptor recovery after experimental detachment and reattachment: an immunocytochemical, morphological, and electrophysiological study. Invest Ophthalmol Vis Sci 2003; 44: 416-425.

17 Kim SD, Nao-i N, Maruiwa F, Sawada A. Electrical responses from locally detached retina and its recovery after reattachment. Ophthalmologica 1996; 210: 195-199.

18 Crossland MD, Culham LE, Rubin GS. Fixation stability and reading speed in patients with newly developed macular disease. Ophthalmol Physiol Opt 2004; 24: 327-333.

19 Crossland MD, Culham LE, Kabanarou SA, Rubin GS Preferred retinal locus development in patients with macular disease. Ophthalmology 2005; 112: 1579-1585.

20 Fletcher DC, Schuchard RA. Preferred retinal loci relationship to macular scotomas in a low-vision population. Ophthalmology 1997; 104: 632-638. 